\title{
High frequency of LOH, MSI and abnormal expression of FHIT in gastric cancer
}

\author{
C. Huiping a , S. Kristjansdottir ${ }^{\mathrm{a}}$, J.T. Bergthorsson ${ }^{\mathrm{a}}$, J.G. Jonasson ${ }^{\mathrm{a}}$, J. Magnusson ${ }^{\mathrm{b}}$, \\ V. Egilsson ${ }^{\mathrm{a}}$, S. Ingvarsson ${ }^{\mathrm{c}, *}$ \\ ${ }^{a}$ Department of Pathology, National University Hospital, Reykjavik, Iceland \\ ${ }^{\mathrm{b}}$ Department of Surgery, National University Hospital, Reykjavik, Iceland \\ ${ }^{\mathrm{c}}$ Institute for Experimental Pathology, Keldur, University of Iceland, 112 Reykjavik, Iceland
}

Received 21 August 2001; received in revised form 19 November 2001; accepted 13 December 2001

\begin{abstract}
The FHIT gene is a putative tumour suppressor gene. In this study, we analysed a set of 50 gastric tumours for alterations of FHIT, and found 38 of 45 tumours $(84 \%)$ exhibiting loss of heterozygosity (LOH) within the FHIT gene. We used both nested Reverse Transcriptase Polymerase Chain Reaction (RT-PCR) and single step RT-PCR to analyse the FHIT transcripts and found 34 of $39(87 \%)$ tumours and seven of the $11(64 \%)$ corresponding non-cancerous tissues showed low or aberrant expression of FHIT mRNA and the appearance of the aberrant FHIT transcripts depended on the conditions of the RT-PCR. In these aberrant transcripts, frequent deletions and/or insertions were detected by direct sequencing. All breakpoints for deletions and insertions were at splicing sites. All insertions came from the adjacent introns, whose appearance was completely in accordance with the 'GUAG' rule for pre-mRNA splicing. It may be suggested that an alternative splicing mechanism functions in the formation of these aberrant transcripts. The fragile nature of FRA3B within the FHIT gene could be responsible for the formation of the aberrant mRNA. Negative or reduced Fhit expression was detected in 39 of 50 tumours (78\%). Moreover, an association was found between abnormal Fhit expression and positive node status $(P=0.012)$. Thirteen of 48 tumours $(27 \%)$ displayed microsatellite instability (MSI), among which 10 tumours also showed MSI within the FHIT gene. Furthermore, we detected an association between MSI and negative node status $(P=0.02)$. We conclude that the abnormalities of $F H I T$, presumably associated with the unstable nature of FRA3B within the FHIT gene, are involved in the carcinogenesis of gastric cancer, and lack of mismatch repair (MMR) could possibly promote its alteration in a subset of gastric tumours. (C) 2002 Elsevier Science Ltd. All rights reserved.
\end{abstract}

Keywords: FHIT; LOH; Mutation; Aberrant mRNA; IHC; MSI; Gastric cancer

\section{Introduction}

The FHIT gene is located at the FRA3B site of chromosome $3 \mathrm{p} 14.2$, and is so far the only example of a relatively well studied gene located in a consititutive fragile region [1]. The FHIT gene is composed of 10 exons which span a $1.8 \mathrm{Mb}$ genome region, of which only exons 5 through to 9 are protein coding. It encodes a small mRNA of $1.1 \mathrm{~kb}$, and a small protein of 16.8 $\mathrm{kDa}$ [2]. Fhit is a homodimer as shown conclusively by crystallographic analysis [3,4]. The Fhit protein is mainly localised in the cytoplasm and has been found in

* Corresponding author. Tel.: +354-567-4700; fax: + 354-5673979.

E-mail address: siguring@ hi.is (S. Ingvarsson). complex with tubulin and ubiquitin conjugating enzyme $[5,6]$. Fhit protein expression is detected in epithelial cells in most human and mouse tissues [1,7]. In higher eukaryotes, Fhit may reduce the intracellular level of diadenosine triphosphate, by binding to it and inducing its hydrolysis [8]. A role of diadenosine triphosphate in the growth control of cells has been suggested $[8,9]$.

Cancer-specific translocations have been mapped within the FHIT gene in renal cell carcinoma and a papilloma virus insertion site in cervical carcinoma [1012]. The breakpoint at $3 \mathrm{p} 14.2$ involved in the $t(3 ; 8)$ chromosome translocation of hereditary renal cell carcinoma, interrupts the third intron of the FHIT gene, inactivating one of the two FHIT alleles [1]. Frequent allelic losses at this region in various malignancies, including gastric cancer, imply that FHIT may represent 
a tumour suppressor gene [2,13]. LOH at the FHIT locus is detected with the highest frequency in familial breast carcinomas lacking functional $B R C A 2$, presumably due to a lack of DNA repair [14-17]. Cell lines from several tumour types, including gastric cancer, carry homozygous deletions at the FHIT locus $[7,11,18,19]$. Abnormal sizes of mRNA have been reported to be tumour-specific in several cancer types, but the role of these low abundance aberrant FHIT transcripts is uncertain $[14,19,20]$. Alternative splicing of the FHIT pre-mRNA has been suggested, but definite characterisation of different splicing products in normal cells has not been carried out and, so far, no alternative proteins have been described [21]. Reduced Fhit expression has been detected in several types of cancers, including gastric cancer, usually as a result of genomic FHIT alterations and altered FHIT transcripts $[2,22-$ 24]. Hypermethylation in the FHIT promoter region has been identified in breast and lung cancer; this is allelespecific and results in reduced expression of the gene [25]. Alterations at the FHIT locus or reduced expression of Fhit have been associated with gastric tumour progression and poor survival of cancer patients [26]. In general, in tumours (lung and stomach) associated with environmental carcinogens, alterations in the FHIT gene occur early in cancer development, but in other cancers are thought to be a late event and possibly associated with cancer progression to more aggressive neoplasias [2]. Somatic point mutations in the FHIT gene have rarely been found in gastric or other tumours so far, and germline mutations do not seem to be significant $[13,19,27]$. Therefore, it has been argued that the FHIT gene may be altered in cancers simply because it is located at a fragile region and is likely to be susceptible to breakage [21].

Various evidence from functional analysis showed the tumour suppressor role of the FHIT gene. Tumorigenicity can be reduced in lung, cervix and kidney cell lines after reintroduction of the FHIT gene [28-30]. Similarly, with the use of human/mouse microcell hybrid, it was demonstrated that the introduction of chromosome 3 into a mouse fibrosarcoma line reduces tumorigenicity, whereas elimination of the FHIT locus enhances tumorigenicity [31]. A definite proof of the tumour suppressor function of Fhit was demonstrated with the generation of Fhit knockout mice [32]. Both heterozygous (Fhit $+/-$ ) and homozygous (Fhit-/-) Fhit knockout mice spontaneously develop tumours at increased frequency [32]. In addition, both Fhit $+/-$ and Fhit $-/-$ mice are more susceptible to carcinogens, and form tumours in the oesophagus and forestomach [32]. Treatment of the Fhit knockout mice with adenovirus and AAV-based expression of the Fhit reduces the malignant phenotype [33]. Apart from the ability of Fhit to bind and hydrolyse Ap3A, little is known of the biochemical and biological function of Fhit with respect to its tumour suppressor ability.

In this study, we analysed a set of 50 gastric tumours for FHIT abnormalities in DNA, mRNA and protein levels in order to clarify the participation of FHIT in gastric carcinogenesis.

\section{Materials and methods}

\subsection{Samples}

Included in the study were 50 tumours and corresponding non-malignant samples, of which two tumours were familial, the rest sporadic. All tumours were available for immunohistochemistry and the majority for DNA and RNA analysis. These cases were diagnosed at the Department of Pathology, University Hospital of Iceland. Fresh tissue was obtained on the day of surgery or from paraffin-embedded material. Information concerning tumour stage, histological type, subtype according to the Lauren classfication [34] and tumour grade was also obtained from the same department. DNA for Polymerase Chain Reaction (PCR) was isolated by the proteinase $\mathrm{K}$ treatment [35]. RNA for Reverse Transcriptase (RT)-PCR was extracted using Tri Reagent (Molecular Research Center, Inc., USA).

\section{2. $\mathrm{LOH}$ determination}

Microsatellite markers used for loss of heterozygosity (LOH) analysis within the FHIT gene were: D3S1313 (telomeric to exon 10), D3S1234 (intron 5), D3S4103 (intron 5), D3S1300 (intron 5), D3S2757 (intron 4) and D3S4260 (intron 4) (Genome Database) (Table 1). The

Table 1

Loss of heterozygosity $(\mathrm{LOH})$ within the FHIT gene in gastric cancer

\begin{tabular}{llllr}
\hline Marker & Location & Number of samples tested & Informative samples (\%) & Tumours with LOH (\%) \\
\hline D3S4260 & Intron 4 & 41 & $16(39)$ & $10(63)$ \\
D3S2757 & Intron 4 & 37 & $20(54)$ & $8(40)$ \\
D3S1300 & Intron 5 & 30 & $26(87)$ & $11(42)$ \\
D3S4103 & Intron 5 & 32 & $26(81)$ & $19(73)$ \\
D3S1234 & Intron 5 & 38 & $27(71)$ & $17(63)$ \\
D3S1313 & Telomeric to exon 10 & 37 & $17(46)$ & $9(53)$ \\
\hline
\end{tabular}


PCR products were separated in an acrylamide sequencing gel and transferred to a positively charged nylon membrane, Hybond-N + (Amersham, Aylesbury, UK) and baked for at least $2 \mathrm{~h}$ at $80^{\circ} \mathrm{C}$. The non-radioactive detection method used to visualise the PCR products has been previously described in Ref. [36]. Autoradiograms were inspected visually by at least two reviewers, comparing the intensity of alleles from normal and tumour DNA. The absence or significant decrease of one allele in the tumour compared with the normal reference sample was considered as LOH.

\subsection{Mutation screening}

Five coding exons (5, 6, 7, 8 and 9) of the FHIT gene were screened for inactivation mutations with a PCRsingle strand conformation polymorphism (SSCP) analysis on genomic DNA templates. The primers for the FHIT gene used in the SSCP analysis were described by Gemma and colleagues (1997), and ordered from TAG Copenhagen A/S. Genomic DNA was used at 30 ng per $25 \mu \mathrm{l}$ reaction mixture containing $5 \mathrm{pmol}$ of the forward and reverse primers, $2.5 \mathrm{nmol}$ of each deoxynucleotide triphosphate (dNTP), 0.5 units of DynaZyme polymerase. The samples were amplified in 35 cycles composed of $30 \mathrm{~s}$ of denaturation at $94^{\circ} \mathrm{C}, 30 \mathrm{~s}$ of annealing at $55{ }^{\circ} \mathrm{C}$, and finally $60 \mathrm{~s}$ of extension at $72{ }^{\circ} \mathrm{C}$. A hot start was used by adding the enzyme in first cycle at approximately $70{ }^{\circ} \mathrm{C}$, after a preincubation time of 5 min at $94{ }^{\circ} \mathrm{C}$. A $4-\mu 1$ aliquot of PCR products was mixed with $7 \mu \mathrm{l}$ of formamide dye $(95 \mathrm{v} / \mathrm{v}$ formamide, $0.05 \mathrm{w} / \mathrm{v}$ bromophenol blue and $0.05 \mathrm{w} / \mathrm{v}$ xylene cyanol), denatured at $94{ }^{\circ} \mathrm{C}$ for $10 \mathrm{~min}$ and snap-cooled on ice. Aliquots of $2 \mu \mathrm{l}$ were analysed simultaneously on two non-denaturing polyacrylamide gels $(5 \mathrm{w} / \mathrm{v}$ acrylamide with $2 \mathrm{w} / \mathrm{v}$ cross-linking), either containing $5 \mathrm{v} / \mathrm{v}$ glycerol or lacking glycerol. Electrophoresis was performed in $1 \times$ Tris Borate EDTA (TBE) on vertical gels at $6 \mathrm{~W}$ overnight or for $6 \mathrm{~h}$ at room temperature. The PCR products were visualised as the microsatellite markers. Samples with abnormal mobility bands were amplified again for 35 cycles as described above. A 5- $\mu$ l aliquot of PCR products was then incubated with $10 \mathrm{U}$ exonuclease I and 2 U shrimp alkaline phosphatase to remove excessive primers and dNTPs (US70995, Amersham). Sequences of both strands were determined by thermo sequenase DNA polymerase (Thermo Sequenase Radiolabeled Terminator Cycle Sequencing Kit, Amersham) using the two original PCR primers.

\subsection{Aberrant $m R N A$ screening}

1-5 $\mu \mathrm{g}$ of the total RNA was reversely transcribed into cDNA in a 15- $\mu 1$ reaction mixture using first strand cDNA synthesis kit (Amersham Pharmacia Biotech). All samples were examined three times using RT-PCR.
One screening was by using non-nested RT-PCR with the expand high fidelity system containing a thermostable Taq DNA polymerase and a proofreading polymerase. This is effective for the amplification of big fragments ( $>500 \mathrm{bp}$ ). By using the expand high fidelity system, the reliability of our experiment can be raised. The remaining two screenings were by semi-nested RTPCR with DynaZyme polymerase. For non-nested RT-

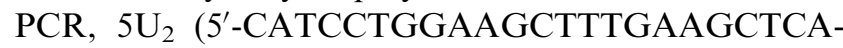
$\left.3^{\prime}\right)$ and 1AS (5'-GCGGTCTTCAAACTGGTTG-3') (731 bp) primers were used [37]. The FHIT cDNA was used at $3 \mu \mathrm{l}$ per $25 \mu \mathrm{l}$ reaction mixture containing 5 pmol of the forward and reverse primers, $2.5 \mathrm{nmol}$ of each dNTP, 2.6 units of high fidelity polymerase. The samples were amplified in 40 cycles composed of $60 \mathrm{~s}$ of denaturation at $95{ }^{\circ} \mathrm{C}, 60 \mathrm{~s}$ of annealing at $60{ }^{\circ} \mathrm{C}$, and finally $90 \mathrm{~s}$ of extension at $72{ }^{\circ} \mathrm{C}$ and a hot start was used as described earlier. For the first step of the nested PCR, 5 $\mathrm{U}_{2}$ and $3 \mathrm{D}_{2}$ (5'-TCACTGGTTGAAGAATACAGG-3') (815 bp) [37] primers were used in the same reaction mixture, but with the DynaZyme polymerase and in the same PCR conditions used in the non-nested RT-PCR, for 30 cycles of amplification. For the second step, $1 \mu \mathrm{l}$ of the first step products was amplified using $5 \mathrm{U}_{2}$ and $1 \mathrm{AS}$ primers, which were identical to the nonnested PCR. The reaction mixture and PCR conditions were the same as the first step of the semi-nested RTPCR, except for the 40 cycles of amplification. Meanwhile, 11 corresponding non-cancerous tissues were also analysed. As internal controls, additional PCR operations were carried out using primers specific for $\beta$-actin (5'-TTCTACAATGAGCTGCGTGT-3', 5'-GGAGTCCATCACGATGCCAG-3', 198 bp), glyceraldehyde-3phosphate dehydrogenase $(G A P D H) \quad\left(5^{\prime}\right.$-AGTCAGCCGCATCTTCTTTTGC-3', 5'-CTCCTGGAAGATGGTGATGGGA-3', 290 bp), and phosphoglycerate kinase $(P G K)\left(5^{\prime}\right.$-GCTGAACTACTTTGCAAAGGC-3', 5-CCCCAGGAAGGACTTTACCT-3', 653 bp) [37]. The PCR products were visualised by $2 \mathrm{w} / \mathrm{v}$ agarose gel electrophoresis. Abnormal fragments were excised and sequenced using forward and reverse primers to determine the boundaries of the deletions and insertions. Here, we used BigDye Terminator Cycle Sequencing Ready Reaction Kit (Perkin-Elmer, Foster City, CA, USA) and automated sequencer ABI PRISM ${ }^{\text {TM }} 3100$ (Perkin-Elmer) for sequencing.

\subsection{Microsatellite instability}

We used 21 polymorphic markers distributed over the genome including the markers used in FHIT $\mathrm{LOH}$ analysis, obtained from Research Genetics: D2S123, D2S382, BAT26, D3S1210, D3S1217, D3S1234, D3S1300, D3S1313，D3S1600，D3S2757，D3S4103，D3S4260, D6S292, D7S480, D9S157, D13S153, D16S421, D16S496, D16S503, D16S512 and D16S545. The MSI + phenotypes 

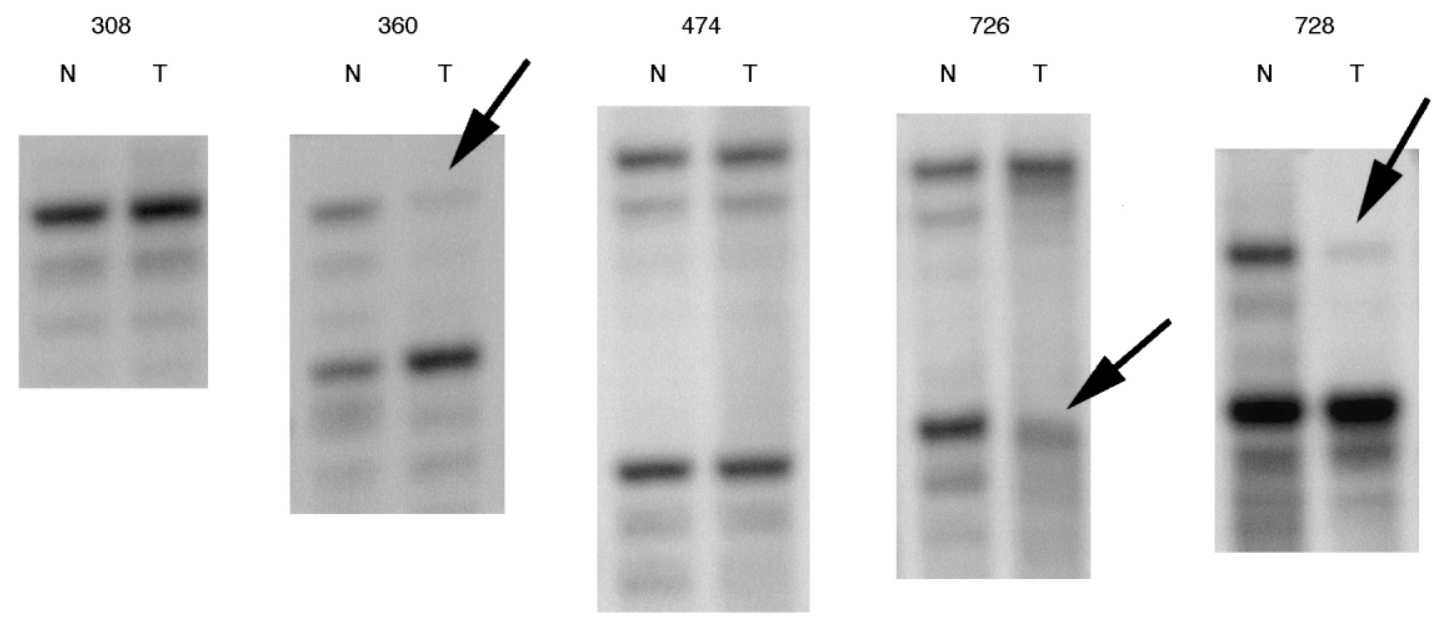

Fig. 1. Autoradiogram of a dinucleotide repeat polymorphism in five matched normal $(\mathrm{N})$ and tumour $(\mathrm{T})$ tissues form patients with gastric cancer. The case numbers are indicated above each autoradiogram. The microsatellite marker D3S1300 was used here. Case 308 is a homozygote. Loss of heterozygosity (LOH) is seen in cases 360,726 and 728 . Arrows point towards alleles with a decrease in relative intensity indicating a LOH. Case 474 is a heterozygote. Each allele has a set of bands due to slippage in the PCR, therefore a homozygote has one set of bands and a heterozygote two sets of bands.

were assigned to tumours where extra alleles were detected at two or more markers.

\subsection{Immunohistochemical staining}

Immunohistochemistry for Fhit was performed on 5 - $\mu \mathrm{m}$ sections from paraffin-embedded tumour tissue blocks with polyclonal antibody glutathione $\mathrm{S}$ transferase (GST)-Fhit. The sections were deparaffinised, rehydrated and rinsed in tap water before antigen retrieval by heating in a $0.01 \mathrm{M}$ citrate buffer $(\mathrm{pH}$ 6.0) twice for 5 $\mathrm{min}$ at $850 \mathrm{~W}$. Sections were incubated with the antibody, diluted $1 / 800$, overnight at room temperature. Immunohistochemical staining was visualised using the Strept ABC Complex/horseradish peroxidase (HRP) Duet (mouse/rabbit from DAKO) according to the manufacturer's instructions. Tumours were graded by the intensity of staining as negative $(-)$, weakly positive $(+)$, moderately positive $(++)$ and strongly positive $(+++)$. A regional variation of staining across the tumours was observed. Therefore, tumours where more than $50 \%$ of the cells were positive for Fhit staining were graded as $+/-,++/-$ and $+++/-$. In contrast,
$-/+,-1++$ and $-1+++$ indicated that more than $50 \%$ of the cells were negative for Fhit staining.

\subsection{Statistical analysis}

A $\chi^{2}$ test or Fisher's Exact test was used to assess the relationship between the above parameters.

\section{Results}

Thirty-eight of 45 tumours (84\%) showed LOH with at least one marker (Fig. 1). Six markers showed high frequency of LOH, namely D3S4103 73\%, D3S4260 $63 \%$, D3S1234 63\%, D3S1313 53\%, D3S1300 42\% and D3S2757 40\%, respectively (Table 1). There were 18 tumours with $\mathrm{LOH}$ at all of the informative markers tested, indicating loss of the whole FHIT gene from one allele in these tumours. The other 20 showed breakpoints within the FHIT gene, suggesting a partial deletion of the FHIT gene in these tumours.

No mutations were detected in the FHIT gene. The polymorphisms detected in the FHIT gene are listed in

Table 2

Polymorphisms of the FHIT gene in gastric cancer

\begin{tabular}{|c|c|c|c|c|c|}
\hline Amplicon & Site & Polymorphism & Frequency $(/ 49)$ & Homozygote/Heterozygote & Somatic/Germline \\
\hline Exon 6 & Codon 54 & GAA (Gln) $\rightarrow$ GAG (Gln) & 1 & $0 / 1$ & $0 / 1$ \\
\hline Exon 6 & Intron 6 & $+43 \mathrm{~A} \rightarrow \mathrm{G}$ & 3 & $3 / 0$ & $0 / 3$ \\
\hline Exon 6 & Intron 6 & $+72 \mathrm{~A} \rightarrow \mathrm{T}$ & 1 & $0 / 1$ & $0 / 1$ \\
\hline Exon 7 & Codon 88 & GCC (Ala) $\rightarrow$ GCT (Ala) & 14 & $1 / 13$ & $0 / 14$ \\
\hline Exon 7 & Codon 92 & GTG (Val) $\rightarrow$ GTA (Val) & 1 & $1 / 0$ & $1 / 0$ \\
\hline Exon 8 & Codon 98 & CAT (His) $\rightarrow$ CAC (His) & 18 & $3 / 15$ & $0 / 18$ \\
\hline
\end{tabular}


Table 2. Here, most of the polymorphisms were heterozygous and from the germline.

Aberrant mRNA, including no bands (low expression), was detected in 34 of 39 tumours $(87 \%)$, and also in seven of 11 corresponding non-cancerous tissues $(64 \%)$. This difference was not statistically significant. Here, the results are the combination of the nested-RTPCR and single step RT-PCR. For nested RT-PCR, 26 of 39 tumours $(67 \%)$ exhibited aberrant mRNA or no bands. In addition, 28 of 39 tumours $(72 \%)$ were found to have aberrant mRNA or no bands using single step RT-PCR with high fidelity polymerase. The accordance rate of the two methods in the samples with visible bands in both methods is $65 \%$. For six tumours with no bands by nested RT-PCR, four showed aberrant mRNA, and the other two wild-type mRNA by single step RT-PCR. In addition, two tumours with no bands by single step RT-PCR were found with aberrant and wild-type mRNA, respectively, by nested RT-PCR. Additionally, all examined samples showed wild-type mRNA in the controls ( $\beta$-actin, GAPDH and $P G K$ ).

We found a different frequency of deletions and insertions in the aberrant mRNA in tumours (Table 3). All breakpoints for these deletions and insertions were at normal or cryptical splicing sites by comparison with the normal sequence of the FHIT gene from a gene bank, in accordance with the 'GU-AG' splicing rule. All insertions came from adjacent introns (Table 3). In particular, two tumours showed insertions that were composed of two sequences from different sites of the same intron (Table 3). Additionally, most of the tumours showed coexpression of wild-type mRNA and aberrant mRNA. However, four tumours had only aberrant mRNA. Interestingly, some tumours displayed two or more aberrant mRNA. However, similar conditions also existed in the corresponding non-cancerous tissues.

Negative $(-)$ or reduced $(+/-,-/+,-/++$ and $-/+++)$ Fhit expression was detected in 39 of 50 (78\%) tumours by immunohistochemistry (Fig. 2).

In addition, we identified MSI in these tumours and found 13 of $48(27 \%)$ showing this phenotype. Interestingly, 10 of 13 tumours (77\%) with MSI also showed MSI within the FHIT gene.

An association was found between absent or reduced Fhit expression and positive node status $(P=0.012$, Fisher's Exact test). No association was found between Fhit expression and tumour stage and grade. Moreover, we found an association between MSI and negative node status ( $P=0.02, \chi^{2}$ test). No association was found between LOH within the FHIT gene and abnormal FHIT transcripts, abnormal FHIT transcripts and
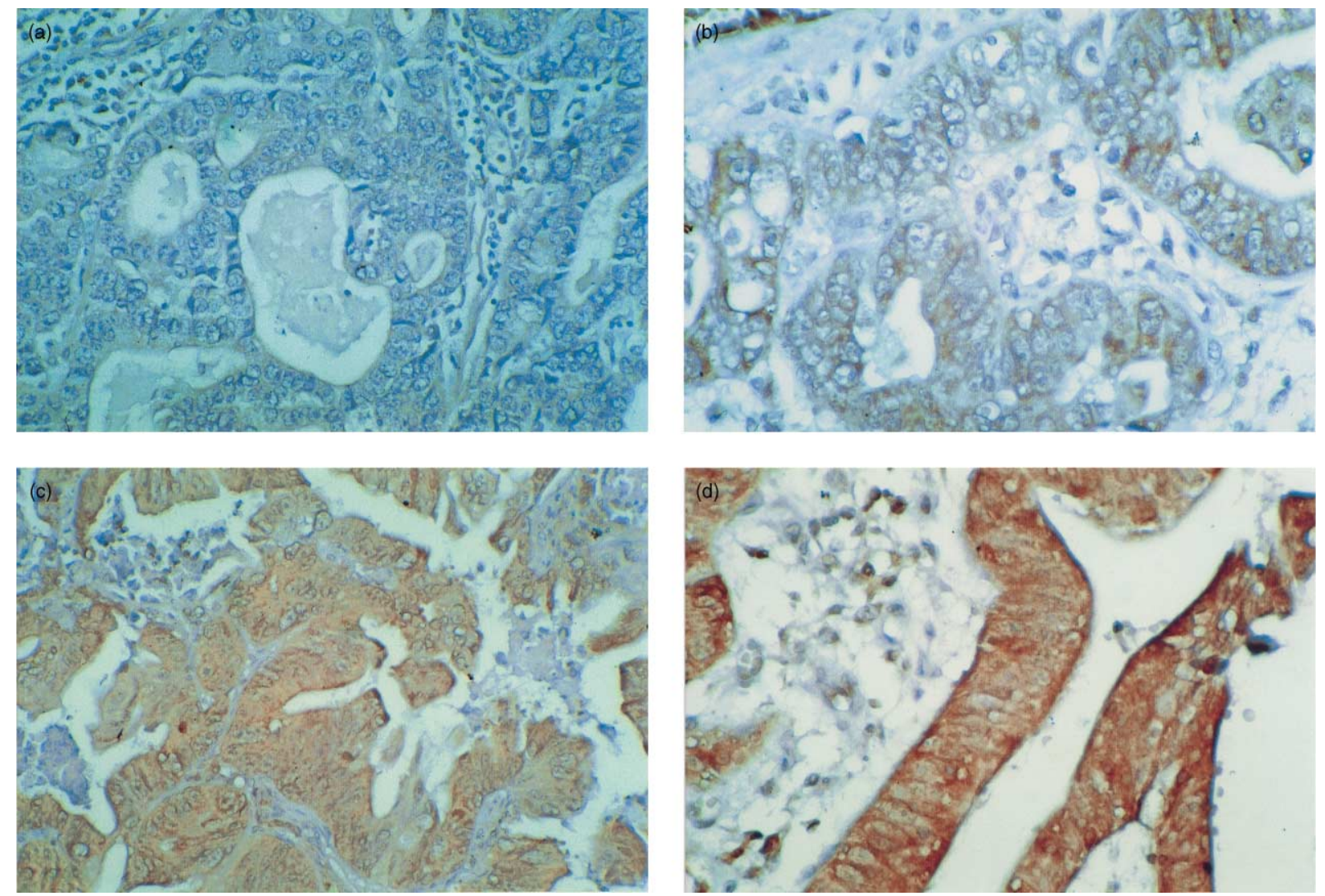

Fig. 2. Immunohistochemical staining of Fhit in intestinal gastric cancers: (a) negative staining (scoring - ); (b) weak staining (scoring + ); (c) moderate staining (scoring ++ ); and (d) strong staining (scoring +++ ). 
Table 3

Analysis of aberrant FHIT transcripts in gastric cancer

\begin{tabular}{ll}
\hline Types of aberration & Frequency (/39) \\
\hline Deletion of exons 3-6 & 15 \\
Deletion of exons 5-8 & 7 \\
Deletion of exons 5-7 & 6 \\
Deletion of ATGTTTTCAG of exon 10 & 5 \\
Deletion of exons 4-7 & 3 \\
Deletion of exons 3-4 & 2 \\
Deletion of exon 4 & 2 \\
Deletion of exons 3-7 & 1 \\
Deletion of exons 4-6 & 1 \\
Insertion of 72 bases between exons 4 and $5^{\mathrm{a}}$ & 3 \\
Insertion of 93 bases between exons 2 and $3^{\mathrm{b}}$ & 1 \\
Insertion of 53 bases between exons 8 and $9^{\mathrm{c}}$ & 1 \\
Insertion of 112 bases between exons 4 and $5^{\mathrm{d}}$ & 1 \\
Insertion of 56 bases between exons 9 and $10^{\mathrm{e}}$ & 1 \\
Insertion of 40 bases between exons 4 and $5^{\mathrm{f}}$ & 1 \\
Deletion of exons $5-7$ and insertion of 138 bases $^{\mathrm{g}}$ & 3 \\
Deletion of exon 3 and insertion of 102 bases $^{\mathrm{h}}$ & 1 \\
Deletion of exons $4-7$ and insertion of 222 bases $^{\mathrm{i}}$ & 1 \\
Deletion of exon 5 and insertion 250 bases & 1 \\
Deletion of exons 5 and 6 and insertion of 86 bases $^{\mathrm{k}}$ & 1
\end{tabular}

All insertion sequences came from adjacent introns, whose appearance is in accordance with the 'GU-AG' rule for pre-mRNA splicing.

a $5^{\prime}$-aaaggacattccaatttacatcctcaccagcatctgtttgggagtgcttctctctctgcattcttgccaact- $3^{\prime}$.

b $5^{\prime}$-cagacagtagaaaaagctgtagccgctgaagagaggccccaaccggaactgtggccttcettagagggatacagccattgccaacccacaatg- $3^{\prime}$.

c $5^{\prime}$-gttttgagaggttcctgtgagccaggccetgggetgggtgcagggacacaagg-3'.

d $5^{\prime}$-gtaccgtgactggatcacatggagccccaggatttttagtaaaggacattccaatttacatcctcaccagcatctgtttgggagtgcttctctctctgcattcttgccaact- $3^{\prime}$.

e $5^{\prime}$-agaagatttctgatgttagaatcataaggcetttgtttgtttaaaaatggcttaag- $3^{\prime}$.

f $5^{\prime}$-gtaccgtgactggatcacatggagccccaggatttttagt- $3^{\prime}$.

g $5^{\prime}$-agtcttgctgtgtcgtccaggctggagtgcagtggtatgatcttggctcactgcaacctctgcetccagggttcaagtgattctgctgcctcagtctcetgagtagctgggactacaggtgtgcgecacaacacccag- $3^{\prime}$, from intron 5 .

h $5^{\prime}$-gcagaaatttcaaggtaaggaagaagaaaagaagaaagggaggaaggaaaggagaagggacggaaaaggattccagtttctgaatttttgtggcatcctact- $3^{\prime}$, from intron 3 .

${ }^{i} 5^{\prime}$-agtcttgctgtgtcgtccaggctggagtgcagtggtatgatcttggctcactgcaacctctgcctccagggttcaagtgattctgctgcctcagtctcctgagtagctgggactacaggtgtgcgecacaacacccagattccaggggtgtgcatccattcttgttacacgggtaaactgagtgtcgctgaagcttggtggatgaatgatcetgtcacccag- $3^{\prime}$, the first 138 bases from one site of intron 5 , the last 84 bases from another site of intron 5 .

j $5^{\prime}$-agtcttgctgtgtcgtccaggctggagtgcagtggtatgatcttggetcactgcaacctctgcctccagggttcaagtgattctgctgcctcagtctcctgagtagctgggactacaggtgtgcgecacaacacccagtctttgagagttcaggccetggtccgagaggattcaatcctgtgggtatgaactgcttggtaagaacactacccaaagccagcagcatgggcettaacagaccttgctacag- $3^{\prime}$, the first 138 bases from middle of intron 5, the last 112 bases was also last 112 bases of intron 5 ,

k $5^{\prime}$-gctctcettcetgctgccatgtaaggtgtgactttgctcttgattcacetggcatgcgtgtgaggcetcctcagccatgtggaact- $3^{\prime}$, from intron 5 .

abnormal Fhit expression, and LOH within the FHIT gene and abnormal Fhit expression.

\section{Discussion}

LOH within the FHIT gene was detected at a high frequency in this study. The microsatellite markers used here were mainly located at introns 4 and 5. However, it is reasonable to deduce that a high frequency of $\mathrm{LOH}$ within the FHIT gene would be identified if markers from other introns were also used. This indicates a highly unstable feature of the FHIT gene in gastric cancer. A common fragile site, FRA3B, was mapped into the FHIT gene [1]. The fragility of this site may presumably result from the late replication, which can be further delayed by inhibiting the DNA polymerase activity by aphidicolin [38]. The common fragile sites are loci that are especially prone to forming gaps or breaks on metaphase chromosomes when cells are cultured under conditions that inhibit DNA replication or repair [39]. In addition to forming fragile sites on metaphase chromosomes, they have been shown to display a number of characteristics of unstable and highly recombinogenic DNA in vitro, including chromosome rearrangements, sister chromatid exchanges and intrachromosomal gene amplification [39]. Therefore, we can speculate that the above aberrations can also occur in these gastric tumours with $\mathrm{LOH}$ within the FHIT gene, if these tumours are generated by carcinogens, which act as inhibitors of DNA replication and repair. However, so far the instability of the fragile sites has not been well documented in relation to tumour initiation and progression. On the other hand, we deduce that the common fragile sites can maintain stability if DNA replication and repair are not inhibited by carcinogens. If so, the high frequency of LOH here strongly suggests that the FHIT gene is a tumour suppressor gene. Loss of one allele of the FHIT gene may cause reduced Fhit expression [23].

Meanwhile, we found $27 \%$ of tumours showing MSI, suggesting that there is a lack of mismatch repair (MMR) in these tumours. This could be partly responsible for the high frequency of $\mathrm{LOH}$ detected within the FHIT gene, by promoting the instability of FRA3B or accumulation of genetic changes in a non-fragile region. Moreover, 10 of 13 tumours (77\%) with MSI also showed MSI in intron 4 or 5 of the FHIT gene, suggesting that introns 4 and 5 are highly unstable. Shortened microsatellite $\mathrm{d}(\mathrm{CA}) 21$ sequence downregulates the promoter activity of the matrix metalloproteinase 9 (MMP9) gene [40]. Therefore, it can be suggested that loss or gain of (CA)n caused by MSI in the FHIT introns could result in reduced expression by affecting pre-mRNA splicing or downregulating the transcriptional activity of the FHIT gene. Additionally, an association was found between MSI and negative node status, suggesting that MSI, caused by lack of MMR, is an initiator in gastric tumorigenesis, which can promote alterations of the FHIT gene or other genes.

The discrepancies in the tumours with visible bands in the two protocols suggest that the appearance of the aberrant mRNA is dependent on the conditions of RTPCR, and the amount of the aberrant mRNA can be 
low. This is in accordance with a previous study in lung cancer [37]. Here, a single step RT-PCR with a highfidelity polymerase was more effective than a nested RTPCR, because in six tumours with no bands by nested RT-PCR, four were shown to have aberrant mRNA and another two to have wild-type mRNA by singlestep RT-PCR. This also indicates that low expression of the FHIT gene occurs in the tumour cells. Previous studies also support this opinion [37]. In the present study, low expression of FHIT mRNA was also detected in the corresponding non-cancerous tissues. Above, we have mentioned that hypermethylation in the FHIT promotor region has been detected in breast and lung cancers; this is allele-specific and results in a low expression of the gene [25]. We continued to sequence these aberrant transcripts by direct sequencing and found frequent deletions and/or insertions in them, but no point mutations were identified. Detailed analysis found that all breakpoints of these deletions and insertions are at normal or cryptical splicing sites, and all insertions came from the adjacent introns by comparison with normal DNA sequences from a gene bank. Interestingly, the appearance of these insertions is completely in line with the 'GU-AG' rule for pre-mRNA splicing. Because no typical DNA mutations at splicing sites were detected, it may be suggested that the formation of these aberrant transcripts is due to alternative splicing. This is supported by the finding that some tumours showed two or more aberrant mRNAs and non-cancerous tissues also exhibited aberrant mRNA. Previous studies also showed aberrant FHIT mRNA in normal tissues [41-44]. The high frequency of alternative splicing of the FHIT mRNA may result from the instability of this locus. It would be of interest to understand better if there is a functional link between the late replication and alternative splicing of this locus. The alternative splicing products are probably not due to dysfunction of the splicesome, because no aberrant transcripts were detected in the controls ( $\beta$-actin, $G A P D H$ and $P G K)$. We did not detect an association of alternative transcripts with reduced Fhit expression, but it should be noted that usually the alternative mRNA is accompanied by wild-type mRNA. It is not clear if these aberrant transcripts affect the gene or cell function, but they could encode structurally abnormal Fhit protein, which may influence the functions of the homodimer Fhit composed of the abnormal Fhit and normal Fhit by dominant-negative mechanisms. However, no alternative Fhit proteins have been described so far.

Negative or reduced expression of Fhit has been detected in $78 \%$ of tumours examined by immunohistochemistry. This frequency is higher than in our previous studies on breast cancer $[23,24]$, suggesting that the alterations of the Fhit proteins may play a major role in a majority of gastric tumours. Presumably absent or reduced Fhit can not modulate the intracellular level of the diadenosine triphosphate, resulting in loss of control of cell growth. Fhit expression also induced apoptosis in studies using human pancreatic and oesophageal cancer cells $[45,46]$. A heterogeneous expression of Fhit was observed across the tumours, suggesting that there are different genetic changes of the FHIT gene in the tumour cells. It is possible that the high frequency of alternative splicing of the FHIT mRNA generates heterogeneous Fhit expression. Additionally, we found an association between abnormal Fhit expression and positive node status, suggesting that alterations of the FHIT gene play a role in the late stage of gastric tumorigenesis. This is accordance with the report by Capuzzi and colleagues [26]. In our previous studies, LOH within the FHIT gene was associated with abnormal Fhit expression in breast cancer $[23,24]$. Therefore, we speculate that $\mathrm{LOH}$ within the FHIT gene could also result in the abnormal expression of Fhit in this study, although a significant association was not found, presumably due to the low numbers in the negative $\mathrm{LOH}$ group.

\section{Acknowledgement}

This work was supported by the University Science Fund and the Icelandic Cancer Society.

\section{References}

1. Ohta M, Inoue H, Cotticelli MG, et al. The FHIT gene, spanning the chromosome 3p14.2 fragile site and renal carcinoma-associated $\mathrm{t}(3 ; 8)$ breakpoint, is abnormal in digestive tract cancers. Cell 1996, 84, 587-597.

2. Croce CM, Sozzi G, Huebner K. Role of FHIT in human cancer. J Clin Oncol 1999, 17, 1618-1624.

3. Lima CD, D'Amico KL, Naday I, et al. MAD analysis of FHIT, a putative human tumor suppressor from the HIT protein family. Structure 1997, 5, 763-774.

4. Pace HC, Garrison PN, Robinson AK, et al. Genetic, biochemical, and crystallographic characterization of Fhit-substrate complexes as the active signaling form of Fhit. Proc Natl Acad Sci USA 1998, 95, 5484-5489.

5. Chaudhuri AR, Khan IA, Prasad V, et al. The tumor suppressor protein Fhit. A novel interaction with tubulin. J Biol Chem 1999, 274, 24378-24382.

6. Shi Y, Zou M, Farid NR, Paterson MC. Association of FHIT (fragile histidine triad), a candidate tumour suppressor gene, with the ubiquitin-conjugating enzyme hUBC9. Biochem J 2000, 352, 443-448.

7. Druck T, Hadaczek P, Fu TB, et al. Structure and expression of the human FHIT gene in normal and tumor cells. Cancer Res 1997, 57, 504-512.

8. Barnes LD, Garrison PN, Siprashvili Z, et al. Fhit, a putative tumor suppressor in humans, is a dinucleoside $5^{\prime}, 5^{\prime \prime \prime}$-P1,P3-triphosphate hydrolase. Biochem 1996, 35, 11529-11535.

9. Murphy GA, Halliday D, McLennan AG. The Fhit tumor suppressor protein regulates the intracellular concentration of diadenosine triphosphate but not diadenosine tetraphosphate. Cancer Res 2000, 60, 2342-2344. 
10. Gemmill RM, West JD, Boldog F, et al. The hereditary renal cell carcinoma $3 ; 8$ translocation fuses FHIT to a patched-related gene, TRC8. Proc Natl Acad Sci USA 1998, 95, 9572-9577.

11. Inoue $\mathrm{H}$, Ishii $\mathrm{H}$, Alder $\mathrm{H}$, et al. Sequence of the FRA3B common fragile region: implications for the mechanism of FHIT deletion. Proc Natl Acad Sci USA 1997, 94, 14584-14589.

12. Wilke CM, Hall BK, Hoge A, et al. FRA3B extends over a broad region and contains a spontaneous HPV16 integration site: direct evidence for the coincidence of viral integration sites and fragile sites. Hum Mol Genet 1996, 5, 187-195.

13. Gemma A, Hagiwara $\mathrm{K}, \mathrm{Ke} \mathrm{Y}$, et al. FHIT mutations in human primary gastric cancer. Cancer Res 1997, 57, 1435-1437.

14. Negrini M, Monaco C, Vorechovsky I, et al. The FHIT gene at 3 p14.2 is abnormal in breast carcinomas. Cancer Res 1996, 56, 3173-3179.

15. Man S, Ellis IO, Sibbering M, et al. High levels of allele loss at the FHIT and ATM genes in non-comedo ductal carcinoma in situ and grade I tubular invasive breast cancers. Cancer Res 1996, 56, 5484-5489.

16. Bergthorsson JT, Johannsdottir J, Jonasdottir A, et al. Chromosome imbalance at the 3 p14 region in human breast tumours: high frequency in patients with inherited predisposition due to BRCA2. Eur J Cancer 1998, 34, 142-147.

17. Ingvarsson $\mathrm{S}$, Geirsdottir EK, Johannesdottir $\mathrm{G}$, et al. High incidence of loss of heterozygosity in breast tumors from carriers of the BRCA2 999del5 mutation. Cancer Res 1998, 58, 44214425 .

18. Kastury K, Baffa R, Druck T, et al. Potential gastrointestinal tumor suppressor locus at the 3p14.2 FRA3B site identified by homozygous deletions in tumor cell lines. Cancer Res 1996, 56, 978-983.

19. Ahmadian M, Wistuba II, Fong KM, et al. Analysis of the FHIT gene and FRA3B region in sporadic breast cancer, preneoplastic lesions, and familial breast cancer probands. Cancer Res 1997, 57, 3664-3668.

20. Hayashi S, Tanimoto K, Hajiro-Nakanishi K, et al. Abnormal FHIT transcripts in human breast carcinomas: a clinicopathological and epidemiological analysis of 61 Japanese cases. Cancer Res 1997, 57, 1981-1985.

21. Ingvarsson S. FHIT alteration in breast cancer. Sem Cancer Biol 2001, 11, 361-366.

22. Baffa R, Veronese ML, Santoro R, et al. Loss of FHIT expression in gastric carcinoma. Cancer Res 1998, 58, 4708-4714.

23. Ingvarsson $\mathrm{S}$, Agnarsson BA, Sigbjornsdottir BI, et al. Reduced Fhit expression in sporadic and BRCA2-linked breast carcinomas. Cancer Res 1999, 59, 2682-2689.

24. Huiping C, Jonasson JG, Agnarsson BA, et al. Analysis of the fragile histidine triad (FHIT) gene in lobular breast cancer. Eur $J$ Cancer 2000, 36, 1552-1557.

25. Zochbauer-Muller S, Fong KM, Maitra A, et al. Frequent occurrence of $5^{\prime} \mathrm{CpG}$ island methylation of the FHIT gene in lung, breast and oral cancer. Cancer Res 2001, 61, 3581-3585.

26. Capuzzi D, Santoro E, Hauck WW, et al. Fhit expression in gastric adenocarcinoma- Correlation with disease stage and survival. Cancer 2000, 88, 24-34.

27. Ingvarsson S, Sigbjornsdottir BI, Huiping $\mathrm{C}$, et al. Alterations of the FHIT gene in breast cancer: association with tumour progression and patient survival. Cancer Det Prev 2001, 25, 318-324.

28. Ji L, Fang B, Yen N, et al. Induction of apoptosis and inhibition of tumorigenicity and tumor growth by adenovirus vector-mediated fragile histidine triad (FHIT) gene overexpression. Cancer Res 1999, 59, 3333-3339.

29. Siprashvili Z, Sozzi G, Barnes LD, et al. Replacement of Fhit in cancer cells suppresses tumorigenicity. Proc Natl Acad Sci USA 1997, 94, 13771-13776.

30. Werner NS, Siprashvili Z, Fong LY, et al. Differential susceptibility of renal carcinoma cell lines to tumor suppression by exogenous Fhit expression. Cancer Res 2000, 60, 2780-2785.

31. Kholodnyuk ID, Szeles A, Yang Y, et al. Inactivation of the human fragile histidine triad gene at 3 p14.2 in monochromosomal human/mouse microcell hybrid-derived severe combined immunodeficient mouse tumors. Cancer Res 2000, 60, 7119-71125.

32. Fong LY, Fidanza V, Zanesi N, et al. Muir-Torre-like syndrome in Fhit-deficient mice. Proc Natl Acad Sci USA 2000, 97, 47424747.

33. Dumon KR, Ishii H, Fong LY, et al. FHIT gene therapy prevents tumor development in Fhit-deficient mice. Proc Natl Acad Sci USA 2001, 98, 3346-3351.

34. Lauren P. The two histological main types of gastric carcinoma: diffuse and so-called intestinal-type carcinoma. An attempt at a histo-clinical classification. Acta Pathol Microbiol Scand 1965, 64, 31-49.

35. Smith SA, Easton DF, Evans DG, Ponder BA. Allele losses in the region 17q12-21 in familial breast and ovarian cancer involve the wild-type chromosome. Nat Genet 1992, 2, 128-131.

36. Vignal A, Gyapay G, Hazan J, et al. Nonradioactive Multiplex Procedure for Genotyping of Microsatellite Markers. Methods in Molecular Genetics, vol. 1. San Diego, Academic Press, 1993, 211-221.

37. Sato H, Hiyama K, Ishioka S, et al. Alternative splicing, but not allelic loss, of the FHIT gene increases with development of lung cancer. Int J Oncol 1999, 15, 81-88.

38. Wang L, Darling J, Zhang JS, et al. Allele-specific late replication and fragility of the most active common fragile site, FRA3B. Hum Molec Genet 1999, 8, 431-437.

39. Glover TW. Instability at chromosomal fragile sites. Rec Res Cancer Res 1998, 154, 185-199.

40. Shimajiri S, Arima N, Tanimoto A, et al. Shortened microsatellite $\mathrm{d}(\mathrm{CA}) 21$ sequence down-regulates promoter activity of matrix metalloproteinase 9 gene. FEBS Lett 1999, 455, 70-74.

41. Wang NM, Chang JG, Liu TC, et al. Aberrant transcripts of FHIT, TSG101 and PTEN/MMAC1 genes in normal peripheral mononuclear cells. Int J Oncol 2000, 16, 75-80.

42. Wang NM, Chang JG. Are aberrant transcripts of FHIT, TSG101, and PTEN/MMAC1 oncogenesis related? Int $J$ Mol Med 1999, 3, 491-495.

43. Tokuchi Y, Kobayashi Y, Hayashi S, et al. Abnormal FHIT transcripts found in both lung cancer and normal lung tissue. Genes Chrom Cancer 1999, 24, 105-111.

44. Yang HW, Piao HY, Taki T, et al. Pattern of FHIT gene expression in normal and leukaemic cells. Int J Cancer 1999, 81, 897-901.

45. Dumon KR, Ishii H, Vecchione A, et al. Fragile histidine triad expression delays tumor development and induces apoptosis in human pancreatic cancer. Cancer Res 2001, 61, 4827-4836.

46. Ishii H, Dumon KR, Vecchione A, et al. Effect of adenoviral transduction of the fragile histidine triad gene into esophageal cancer cells. Cancer Res 2001, 61, 1578-1584. 